Cinémas

Revue d'études cinématographiques

Journal of Film Studies

\title{
Au-delà du cinéma. Image et temps numériques
}

\section{Edmond Couchot}

Volume 5, numéro 1-2, automne 1994

Le Temps au cinéma

URI : https://id.erudit.org/iderudit/1001005ar

DOI : https://doi.org/10.7202/1001005ar

Aller au sommaire du numéro

Éditeur(s)

Cinémas

ISSN

1181-6945 (imprimé)

1705-6500 (numérique)

Découvrir la revue

Citer cet article

Couchot, E. (1994). Au-delà du cinéma. Image et temps numériques. Cinémas, 5(1-2), 69-80. https://doi.org/10.7202/1001005ar

\section{Résumé de l'article}

L'auteur, en retraçant les statuts temporels du cinéma (re-présentation), des émissions en " direct » (sur-présentation), avance l'idée que l'image numérique (simulation) se distingue essentiellement de celle du cinéma et de la télévision par son caractère dialogique ou interactif. Sont ici mises au jeu deux grandes catégories de temps relatives à l'image, le temps du voir et le temps $d u$ faire. L'auteur interroge la technologie numérique en tant qu'elle peut être représentative d'une nouvelle esthétique dans laquelle le temps est uchronique et où l'acte de figuration complet n'a lieu que par la présence du regardeur : le temps du voir est aussi, autrement et à la fois, un temps du faire. 


\section{Au-delà du cinéma. Image et temps numériques}

\section{Edmond Couchot}

\section{RÉSUMÉ}

L'auteur, en retraçant les statuts temporels du cinéma (re-présentation), des émissions en "direct " (sur-présentation), avance l'idée que l'image numérique (simulation) se distingue essentiellement de celle du cinéma et de la télévision par son caractère dialogique ou interactif. Sont ici mises au jeu deux grandes catégories de temps relatives à l'image, le temps $d u$ voir et le temps $d u$ faire. L'auteur interroge la technologie numérique en tant qu'elle peut être représentative d'une nouvelle esthétique dans laquelle le temps est uchronique et où l'acte de figuration complet n'a lieu que par la présence du regardeur: le temps du voir est aussi, autrement et à la fois, un temps du faire.

\section{ABSTRACT}

Through a review of the temporal statuses of cinema (re-presentation) and of live broadcasts (over-presentation), the author suggests that digital imaging (simulation) is essentially different from both cinema and television because of its dialogic or interactive character. Two broad temporal categories enter into the discussion: the time of seeing and the time of making. The author examines digital technology as a representative of a new aesthetic in which time is achronic and in which the act of figuration remains incomplete without the presence of the viewer: the time of seeing is also, in a different way and concurrently, the time of making. 
Il est difficile d'aborder la notion de temps dans son rapport à l'image (fixe ou mobile), sans mêler assez confusément le technique et le subjectif, les matériaux figuratifs constituant l'image et son interprétation. Pour la simplifier, je proposerai de distinguer deux grandes catégories de temps relatives à l'image.

D'abord, tout en aval de l'image, de son devenir, le temps $d u$ voir. C'est le temps où l'image est effectivement perçue par le regardeur, où elle se présente à lui : temps objectivement analysable (du moins relativement) de la distribution de l'image (sa mise en circulation, ou encore sa médiation, et plus généralement, sa socialisation), soumis à ses propres lois locales et circonstancielles, hors duquel nulle image n'est visible et qui en module très fortement le sens et les effets. Mais le temps du voir est aussi, par excellence, le temps, subjectif, celui-là, du regard, des effets sur la perception visuelle, du mouvement et de l'itinéraire des yeux sur la surface de l'image, de sa lecture et de son interprétation. Il est propre au regardeur et il est activé par lui, mais il est aussi orienté plus ou moins fortement par les éléments figuratifs constituant l'image et la culture.

À l'inverse, et cette fois-ci en amont de l'image, le temps $d u$ faire. Il correspond à la durée, brève ou longue, au cours de laquelle l'image est engendrée. En photographie, il se condense presque exclusivement dans l'instant de la pose. En peinture, il s'étend plus largement. Le temps du faire est en grande partie le temps où les techniques figuratives sont à l'œuvre. Or, le faire technique n'est pas un simple moyen d'exécution qui ne ferait que traduire ce que l'imagination a auparavant conçu. C'est aussi une façon de percevoir le monde, l'espace et le temps en particulier, et d'en offrir des représentations, certes parcellaires et éclatées, mais déjà soumises à une certaine logique. Si bien que l'expérience perceptive qui accompagne la réalisation concrète de toute image donne à son créateur l'occasion de vivre des temporalités très remarquables et propres aux techniques. Ces temporalités se transmettent de l'«imageur" au "regardeur", pendant le temps du voir, et participent, elles aussi, à la dimension temporelle de l'image vue. Le temps du faire n'est pas seulement le temps de la technique et de ses processus, c'est aussi le temps où l'imageur conçoit et exprime une intention figurative 
(individuelle ou collective), où s'élabore la pensée figurative. Le temps du faire se condense en un acte décisif, l'acte de figuration: moment de la naissance au monde et au temps de l'image '

\section{Une adhérence au temps}

Au cinéma, le temps du faire se décompose en deux phases essentielles. Celle de la prise de vue et celle du montage. La prise de vue est, comme dans la photo, inséparable de la prise de temps, mais cette prise de temps n'est plus réduite au bref instant de la pose; elle englobe des suites organisées d'instants. Elle n'est plus ponctuelle mais étendue, séquentielle, composée d'une succession de photogrammes - le plan. Cependant, dans le cinéma, comme le remarque Gilles Deleuze ${ }^{2}$, les instants séparant les photogrammes ne sont plus des instants privilégiés comme en photographie, choisis le plus souvent avec une extrême attention, ils sont déterminés par un automatisme qui se limite à contrôler rigoureusement mais aveuglément les intervalles temporels séparant chaque arrêt de l'image. En revanche, si le temps d'exposition de chaque photogramme n'est plus décisif, comme la pose en photographie, c'est le plan et sa propre durée, choisie, elle, avec tout le soin désiré, qui le deviennent.

Mais le temps du faire cinématographique ne se réduit pas aux mécanismes de la prise de vue et de la prise de temps. Il faut encore et surtout couper, organiser, enchaîner les plans. Grâce au montage, le cinéaste recrée un ordre différent qui réassemble les plans et leur redonne un sens temporel. Opération axiale par laquelle s'insinuent sa subjectivité et sa propre temporalité, l'œuvre s'autonomise et se déprend du réel. Ces deux opérations techniques constituent l'essentiel de l'acte de figuration cinématographique, à la suite duquel naît l'œuvre filmique, et cela depuis le début du cinéma. La technique a, en effet, peu évolué. Le son, la couleur, les grands formats n'ont rien changé au principe d'enregistrement photomécanique de l'image filmée et à l'assemblage linéaire des plans. Toutefois, cette stabilité technique et ses contraintes ont largement contribué à en faire un art complet, avec ses styles, ses écoles, ses traditions et ses révolutions.

Le temps du voir au cinéma est socialement très contrôlé. La salle de projection doit isoler les spectateurs du monde environ- 
nant, rabattre les regards sur l'écran. Tous convergent vers la même image. Tous les yeux occupent en même temps la même place derrière la caméra, ou devant la visionneuse, le lieu même où s'est tenu à l'origine, c'est-à-dire pendant le temps du faire, l'œil du réalisateur. (Il y a là un effet puissant de synchronisation et de massification, de "mise en forme de la perception", de "résonance", que ni la peinture, ni la photo, ni même les arts de la scène ne sauraient avoir). Lts spectateurs partagent le même temps filmique, ils le vivent ensemble, à l'unisson. Chaque plan du film plonge le regardeur dans l'écoulement d'une durée originaire, préexistante, et fait coïncider l'empreinte de cette durée avec sa propre durée intérieure, son temps subjectif. Tandis que la photographie plaque le regardeur sur le réel au moyen d'un faisceau de lumière immobilisé - qu'elle le "fait adhérer" au réel, disait Barthes —, le cinéma le rabat sur une autre réalité, celle de la durée, au moyen d'un faisceau de temps, non pas d'une façon abstraite et générale, mais singulière et unique. Elle le rabat sur son écoulement événementiel, jamais reproductible intégralement (dix prises de la même scène ne sont jamais identiques), sur son irréversibilité ${ }^{3}$.

Techniquement, le cinéma fait adhérer le regardeur au temps. Mais à un temps - à un présent du temps — qui a déjà été, qui a déjà été vécu réellement par les acteurs, par les choses filmées, par le réalisateur et son équipe. Ce présent-là ne se reproduira plus jamais. Il ne se représentera plus que sous l'aspect d'un enregistrement. Le film reproduit ce présent lors de la projection, il le re-présente. L'art cinématographique consistera à jouer sur cette adhérence au temps et, par le biais du montage en particulier, à la rendre soit plus forte (tout le phénomène d'identification s'y enracine) ou, au contraire, à s'en déprendre (effets de distanciation, placage sur des temporalités beaucoup plus subjectives, imaginaires, non réalistes, autrement croisées, etc.). Le sens d'un film n'est accessible qu'à la condition que le spectateur puisse remonter jusqu'au temps du faire et le partager avec l'auteur et que s'établisse une certaine résonance, une mise en phase, entre le temps du voir et le temps du faire. 


\section{Sur-présentation}

Avec la télévision, le temps du faire et le temps du voir se présentent différemment. D'un point de vue temporel, la caractéristique essentielle de l'image électronique est d'être vue au moment même où elle est filmée. Dans les émissions en "direct ", la saisie de l'image en mouvement, sa transmission et sa réception sont simultanées. Le direct fait coïncider, sans aucun décalage perceptible, le moment précis où l'image est engendrée et celui où elle est perçue. Il superpose, il incruste l'une dans l'autre deux temporalités: le présent des choses filmées par la caméra, vécu également par celui qui filme, et le présent du regardeur, et cela, indépendamment de la distance. D'où, l'impossibilité du montage et de tout travail de reconstruction temporel ${ }^{4}$. L'acte de figuration télévisuelle tient tout entier dans la saisie directe des images. Tandis que la photo et le cinéma renvoient à un "ça-a-été" imposé par l'enregistrement, la télévision renvoie à un "c'est " immédiat et brutal. Cette collusion violente confond dans le même présent le temps du faire et le temps du voir en les court-circuitant. L'accès au sens de l'image ne se fait qu'à la condition que le temps du faire coïncide sans aucun délai avec le temps du voir. L'image en direct nous plonge ainsi dans un perpétuel présent. Rien n'y prend sens que par rapport à ce présent sans cesse renouvelé, ce placage sur l'événement, l'actualité, au détriment du passé et du futur. Le temps du direct est unidimensionnel. Il n'y a plus re-présentation, distanciation, comme dans la photo et le cinéma, mais sur-présentation. La télévision ne fonctionne pas continuellement sur le principe du direct, mais c'est bien lui qui lui donne toute sa force et sa fascination. Les moments clés de la télévision (journaux, débats, grandes émissions) sont généralement en direct. Et ce sera contre ces contraintes, ou en en jouant, que se construira l'art vidéo, qu'il travaille sur l'image elle-même ou sur ses environnements.

\section{Le mode dialogique}

La technologie numérique introduit, quant à elle, une rupture radicale dans les rapports de l'image au temps. Elle fait passer l'image du mode de la re-présentation/sur-présentation au mode de la simulation. Encore faut-il bien préciser de quel type 
d'images numériques on parle. S'il s'agit des images de synthèse utilisées pour les effets spéciaux du cinéma (du genre Terminator ou Jurassic Park), rien ne change dans leur rapport au temps puisqu'elles sont intégrées à la pellicule; elles épousent le temps du cinéma. En revanche, beaucoup de choses changent dans la manière de produire ces images (les rapports du réalisateur à l'image ne sont plus exactement ceux du cinéma ou de la photographie, encore moins de la peinture) ainsi que dans la perception des formes nouvelles qu'elles donnent à voir. Mais je ne m'attarderai pas sur ce point et je m'en tiendrai à ce qui constitue la spécificité technique de l'image numérique dans son rapport au temps. L'image numérique se distingue essentiellement de celle du cinéma et de la télévision par son caractère dialogique - ou interactif: elle est capable de réagir aux sollicitations diverses du regardeur, bref de lui "répondre" plus ou moins rapidement. Ce dernier peut engager avec l'image un véritable dialogue visuel, allant du très simple au très complexe. Les dispositifs qui autorisent le mode dialogique sont de plus en plus nombreux et diversifiés. Certains permettent d'engendrer des images, en deux ou en trois dimensions, avec lesquelles, quand les conditions sont requises, le regardeur peut interagir en "temps réel ", c'est-à-dire quasi immédiatement, sans utiliser le clavier de l'ordinateur (au moyen, par exemple, de souris, de volant, de manche à balai, de gants et de vêtement de données, etc.).

Limitée à l'origine à quelques utilisations rares et coûteuses, réservées au domaine militaire et aéronautique, cette technologie se répand, se diversifie et se banalise de plus en plus. Elle a pénétré largement le domaine des jeux - jeux d'échecs et de dames électroniques, mais aussi jeux de rôles ou d'action, jeux d'arcades, jeux pédagogiques, etc. (il existe 80 millions de consoles actuellement dans le monde) - , ainsi que les sports (tennis, golf ou équitation sur écran électronique, etc.). L'édition propose de plus en plus de produits interactifs sous forme de vidéodisques ou de CD-I (compact disc interactive) assez élaborés. Les musées commencent à utiliser des banques de données interactives, sous l'aspect d'images projetées sur écran, pour présenter leurs réserves sans que le spectateur soit obligé de se 
déplacer. Dans le domaine des télécommunications, il devient possible, grâce aux techniques dites de "téléprésence" ou de "télévirtualité", de réunir deux ou plusieurs personnes dans un lieu virtuel où aucune d'entre elles n'a accès, comme si elles étaient réellement présentes, ou encore d'agir à distance dans des milieux dangereux ou inaccessibles. Mais si ces techniques sont encore expérimentales et vouées à un futur assez lointain, le multimédia en revanche, en associant la télévision, le téléphone et l'ordinateur, propose à un public beaucoup plus large d'accéder interactivement, et sans attendre demain, à des banques de données diverses et quasi illimitées. La bataille économique, culturelle et politique dont cette industrie naissante est actuellement l'enjeu est la preuve incontestable de la très grande importance qu'elle va prendre dans les années à venir.

D'autres industries, et fort nombreuses, ont aussi adopté sans réserve cette technologie: l'aéronautique, qui a été la première à utiliser des environnements virtuels interactifs pour la formation des pilotes au sol (les bien connus simulateurs de vol), l'automobile, la robotique, et d'une manière générale toutes les industries qui utilisent la CAO, comme le design, l'architecture ou l'urbanisme. Les exemples d'utilisation de l'interactivité numérique dans les sciences et leurs applications sont également nombreux : de la chirurgie à l'astrophysique (où l'expérimentateur reconstitue virtuellement des univers entiers en formation et qu'il soumet à ses hypothèses), en passant par la chimie organique (où l'on combine des molécules virtuelles géantes en les déplaçant de ses propres mains), les études de croissance des végétaux en temps accéléré, et bien d'autres phénomènes. Un nouveau type d'expérimentation - l'expérimentation numérique -, de plus en plus répandue dans le domaine scientifique, offre la possibilité de réinitialiser indéfiniment les processus de calcul utilisés dans la simulation de modèles dont on veut éprouver la validité.

\section{Temps uchronique}

Toutes ces techniques placent le regardeur dans une situation dialogique par rapport à l'image et lui font vivre une expérience perceptive sans précédent de l'espace et du temps. Il semblerait même que les bouleversements d'ordre temporel aient des 
conséquences d'autant plus importantes qu'ils sont inattendus et qu'ils n'appartiennent pas à l'ordre du visible. Le temps du faire et le temps du voir numériques entretiennent de ce fait des relations totalement différentes. Il faut d'abord remarquer que le temps de l'ordinateur est, à l'instar des images qu'il engendre, entièrement synthétisé à partir des micropulsations scandant la marche des opérations de calcul. À la synthèse de l'image correspond une synthèse du temps où le temps, désormais simulé, n'est plus à l'image d'un temps cosmique universel, entraînant les êtres et le monde dans son flot irréversible et les soumettant au même devenir, aux mêmes repères, à la même chronicité. Il n'a plus rien de commun avec le temps scandé par l' "horloge" de la caméra de cinéma ou par le balayage de la double trame de l'écran électronique. Le temps de synthèse est un temps uchronique. Alors que la photographie et le cinéma enregistrent le temps accompli de l'objet qui passe devant l'objectif, et que la télévision, dans le cas du direct, saisit le temps en train de s'accomplir de ce même objet, l'image numérique, elle, du fait qu'elle ne résulte d'aucun enregistrement - puisque aucun objet réel ne lui préexiste - ne donne ni à revivre un présent déjà vécu ni à vivre un présent en train d'être vécu. Elle en engendre un autre qui n'a jamais été et qui ne se répétera jamais systématiquement. Elle renvoie à des multitudes de présents, tous originaires, mais plus ou moins probables, susceptibles éventuellement de s'actualiser sur l'écran. Le temps du faire qui la caractérise ne relève ni d'un "ça-a-été " se référant au passé, ni d'un "c'est" renvoyant à un présent perpétuel, mais d'un "çapeut-être ", ouvert à d'innombrables éventualités.

À cela s'ajoutent encore les effets du temps réel. Le déroulement des processus de calcul, du fait que le regardeur y accède, peut être interrompu ou modifié à chaque instant, échapper à son propre devenir programmatique. Chaque intervention du regardeur (dans certains cas, le seul déplacement de ses yeux) fournit à l'ordinateur un flux de données qu'il interprète et qu'il traduit quasi instantanément. L'image que le regardeur perçoit n'existe donc que dans la mesure où elle est liée à son regard et à son action. Il en va de même pour sa temporalité. Elle résulte de l'interfaçage qui s'établit entre le temps du regardeur, celui qu'il 
vit au moment où il voit l'image, et le temps de l'image au moment où elle est engendrée par le calcul. Dans la situation dialogique, le temps machinique du calcul s'hybride étroitement avec le temps existentiel, singulier et non réitérable de ce dernier. Tout se passe comme si, pendant le temps très court où la machine cérébrale du regardeur est occupée à traiter l'image avec laquelle il dialogue - le bref instant où se régénère l'activité des récepteurs rétiniens — , s'engouffrait, comme en une brèche secrète ouverte dans l'épaisseur de la perception, un supplément de temps au cours duquel d'autres éventualités se préparent. Tandis qu'au cinéma (à la télévision, on se trouve à peu près dans la même situation), l'intervalle de temps séparant deux photogrammes successifs n'est occupé que par la brève durée, imperceptible, de l'obturation, le même intervalle de temps, quand il s'agit d'une image interactive calculée en temps réel, est occupé, comblé, par des millions d'opérations logiques qui résultent en partie de l'intervention du regardeur et qui modifient la réalité simulée. Tout se passe comme si, en définitive, du temps s'ajoutait au temps. Mais un temps de synthèse, tissé de calculs et d'opérations logiques.

Utiliser une cuisine avant qu'elle ne soit construite, en manipulant des images - comme nous y invitent des architectes japonais - , piloter un avion avant qu'il n'ait réellement décollé, vérifier des hypothèses sur la création de l'univers en les soumettant à des expériences virtuelles au cours desquelles le monde naîtra et renaîtra indéfiniment comme si le temps était remis à zéro au gré de l'expérimentateur, c'est faire et défaire les figures du temps, non pas mentalement comme pendant la projection d'un film, mais réellement, même si cette réalité n'est que virtuelle. Comme le temps enregistré du cinéma, le temps uchronique peut être accéléré, ralenti, inversé, renvoyé à son origine pour être lu une nouvelle fois, mais - ce qui est autre chose il peut emprunter des itinéraires différents, souvent imprévus, qui dans certaines circonstances sont pour le regardeur des découvertes et des expériences à vivre inattendues. Le temps de synthèse ne renvoie plus à des événements mais à des éventualités, à un devenir possible du calcul, dépendant du devenir même de l'image, des programmes qui l'engendrent et des réactions du 
regardeur. En sorte que, de même que l'espace ne nous parvient plus qu'à travers une matrice mathématique qui en transforme et réinterprète chaque point lumineux, de même, le temps ne nous est plus perceptible qu'à travers le fractionnement en nanosecondes du calcul qui remplit, à notre insu, le temps mort de notre perception. Entre le temps "vrai" du monde et notre temps subjectif s'interpose désormais ce qu'on nomme paradoxalement le temps réel, comme s'il n'y avait plus de réel que ce temps-là.

\section{Du côté de l'art}

Les possibilités de l'image interactive en temps réel n'ont pas manqué d'intéresser fortement les artistes - musiciens et plasticiens. Très coûteuse, surtout quand les conditions de l'«immersion" dans l'image visent un maximum d'effets en temps réel et exigent l'utilisation de "visiocasques", de vêtements de données, de fauteuils électroniques, de dispositifs "à retour d'effort ", etc., cette technologie reste dans l'ensemble peu accessible, mais déjà des plasticiens s'en sont saisis. Du côté de la musique, les créateurs ont plus de facilité à manipuler des sons et des instruments virtuels et ils ne s'en sont pas privés non plus. J'ai réalisé moimême en 1988, avec la collaboration de Michel Bret et MarieHélène Tramus, tous deux enseignants à l'Université de Paris VIII, un dispositif interactif qui exploitait pour la première fois le souffle comme moyen d'interaction avec une image. Au bas de l'écran d'un processeur graphique repose une petite plume d'oiseau. Le jeu consiste à souffler dessus. Dès qu'un léger souffle atteint la plume, celle-ci s'élève plus ou moins rapidement, avec des mouvements variés dépendant de la force et de la durée du souffle. Lorsque le souffle cesse, la plume retombe en suivant des trajectoires complexes, chaque fois différentes. Si le souffle est trop fort ou trop long, la plume sort complètement du champ de l'écran. Il faut alors attendre quelques secondes pour la voir réapparaître, tombant doucement du haut du cadre. Une autre version de ce dispositif donne à voir une fleur de pissenlit, souvenir d'un slogan fameux: "Je sème à tout vent." Lorsque l'on souffle sur la fleur, des multitudes d'akènes s'en détachent et s'en vont au hasard du vent. Chacun effeuille la sphère étoilée 
à sa manière, rapidement ou lentement, cherchant le rêve ou l'efficacité. Certains, parfois, ne comprennent pas tout de suite qu'il s'agit d'une simulation et croient que les images sont déjà enregistrées. Ces images n'existent pourtant que dans la mesure où le souffle du regardeur les fait naître au moment précis où sa propre temporalité d'être singulier s'hybride au temps uchronique des processus de synthèse. L'acte de figuration complet n'a lieu que par la présence du regardeur, par l'interaction de son corps avec le déroulement des programmes.

\section{La question de l'auteur}

L'acte de figuration numérique, sitôt que le regardeur a la possibilité d'interagir avec l'image, devient donc très différent de ce qu'il est dans le cinéma et la télévision. On ne saurait le réduire à l'écriture des programmes, tout en amont, car cette écriture à elle seule ne produit pas l'image. On ne saurait le réduire à la seule action du regardeur. Il a ceci de singulier qu'il mêle et associe intimement, dans une résonance immédiate et puissante, le temps du voir et le temps du faire, puisque voir l'image, c'est ausi la faire. Ce nouvel état du regard et de l'image ouvre des territoires inexplorés aux arts et à la recherche et pose de multiples questions.

Je n'en évoquerai qu'une, mais capitale, celle de l'auteur. N'importe quelle peinture, n'importe quelle photo, n'importe quel film, n'importe quelle émission de télévision (du type direct) — même totalement dépourvus d'intérêt artistique ont un auteur. Quelqu'un, en amont de l'image, maniant un pinceau, un appareil-photo ou une caméra, a fait l'image et l'a faite seul, du moins sans que le regardeur intervienne à aucun moment. Les images dialogiques (ou les sons), elles, ont deux auteurs: le premier est à l'origine non de l'image, mais du processus de calcul automatique engendrant l'image. Toutefois, sans l'autre auteur - auteur d'ailleurs potentiellement innombrable - et à qui est destinée, en aval, l'image, il n'y a pas d'image. Peut-être, dans ce cas, le terme d'auteur est-il trop fort? À considérer ce qui se fait aujourd'hui, où la part de responsabilité du regardeur est étroite, souvent réduite à de simples manipulations mécaniques, cela est vrai. Mais rien n'empêche d'imaginer, et 
pas forcément sur un mode ludique où la seule possibilité est de gagner ou de perdre, que cette responsabilité puisse être de plus en plus complexe. Il faudrait alors imaginer une autre esthétique où le faire et le voir échangent d'intenses et fécondes relations. Cette esthétique ne serait d'ailleurs pas absolument nouvelle, au demeurant, puisqu'elle s'inscrirait, mais avec d'autres moyens, dans une ligne que de nombreux artistes et théoriciens ont déjà ébauchée (l'œuvre ouverte, la participation du spectateur, etc.). Je n'y vois pas forcément — rassurez-vous — l'au-delà du cinéma. Non pas parce que les tentatives actuelles d'un cinéma interactif seraient peu convaincantes (en fait, elles le sont ${ }^{5}$ ), mais surtout parce que la dimension temporelle du cinéma est d'une nature entièrement différente de celle de l'image dialogique.

\section{Université de Paris VIII}

\section{NOTES}

1 Voir sur cette question mon article, "Résonance - La condition d'intelligibilité de l'image ", Littérature, n 87 (octobre 1992).

2 Voir notamment Cinéma 1. L'image-mouvement (Paris: Minuit, 1983) p. 7-22.

3 En inversant l'écoulement événementiel du film, on ne peut qu'inverser le sens du film. Le ralentissement ou l'accélération n'apportent que des transformations analogiques aux durées originaires.

4 Il peut y avoir, bien sûr, montage d'images différentes, mais elles sont toujours filmées au même moment, ou encore un commentaire qui tendra à se substituer à cet impossible montage temporel.

5 On ne saurait le leur reprocher, sachant après tout que le cinéma a commencé de la même façon, dans les foires. 